\title{
Fanny LIGNON (dir.), Genre et jeux vidéo
}

Toulouse, Presses universitaires du Midi, coll. « Le Temps du Genre», 2015, 268 p.

Simon Massei

\section{(2) OpenEdition}

Journals

Édition électronique

URL : http://journals.openedition.org/clio/13056

DOI : 10.4000/clio.13056

ISSN : 1777-5299

Éditeur

Belin

Édition imprimée

Date de publication : 1 juin 2016

ISBN : 978-2-7011-9852-1

ISSN : 1252-7017

\section{Référence électronique}

Simon Massei, «Fanny Lignon (dir.), Genre et jeux vidéo », Clio. Femmes, Genre, Histoire [En ligne], 43|

2016, mis en ligne le 07 juillet 2016, consulté le 23 septembre 2020. URL : http://

journals.openedition.org/clio/13056; DOI : https://doi.org/10.4000/clio.13056

Ce document a été généré automatiquement le 23 septembre 2020.

Tous droits réservés 


\section{Fanny LIGNON (dir.), Genre et jeux vidéo}

Toulouse, Presses universitaires du Midi, coll. « Le Temps du Genre », 2015, 268 p.

\section{Simon Massei}

\section{RÉFÉRENCE}

Fanny LIGNON (dir.), Genre et jeux vidéo, Toulouse, Presses universitaires du Midi, coll. «Le Temps du Genre », 2015, 268 p.

1 Résultat très attendu d'un colloque organisé en juin 2012 auquel il emprunte son titre, Genre et jeux vidéo rassemble les contributions de quelques quatorze auteur-e-s de différentes disciplines et nationalités. Dirigé par Fanny Lignon, cet ouvrage pionnier met à la disposition du public francophone un premier bilan des travaux réalisés sur un sujet bien souvent déconsidéré dans le champ universitaire car situé à l'intersection de domaines de recherche à faible légitimité.

2 L'ouvrage se divise en deux parties thématiques introduites chacune par un " générique » de cadrage. La première d'entre elles, consacrée aux imaginaires genrés imprégnant les jeux vidéo, s'ouvre sur une réflexion épistémologique convaincante, nourrie de la ciné-psychanalyse de la théoricienne féministe américaine Laura Mulvey, dans laquelle Mathieu Triclot, philosophe spécialiste des jeux vidéo, cherche à « distinguer plusieurs voies possibles pour situer la performance du genre dans les actes de jeu » (p. 19). Les différents chapitres de cette première partie, principalement fondée sur des études de contenus et/ou de l'expérience de jeu (gameplay), offrent par la suite une série de portraits nuancés de personnages de jeux vidéo majoritairement féminins.

3 La contribution d'Alexis Blanchet, traitant dans une démarche diachronique des différentes vies - vidéoludiques, cinématographiques, littéraires, publicitaires et graphiques - de «l'archétype » Lara Croft (p.37), refuse ainsi « de trancher 
définitivement sur sa dimension sexiste ou libératrice»(p.51); elle engage au contraire à voir dans l'héroïne de Tomb Raider "une entité protéiforme, objet de variations multiples et de réceptions différentes » (p. 50).

4 Le chapitre suivant, signé Marion Coville et consacré à trois héroïnes de jeux vidéo, s'efforce similairement de voir en la sorcière Bayonetta davantage qu'une accumulation d'éléments féminins stéréotypés et démontre toute l'ambivalence du personnage sans pour autant prêter d'intentions féministes à ses créateurs masculins.

Bien que le lien avec les questionnements canoniques des études de genre semble plus ténu dans les chapitres 3 et 4, portant respectivement sur la saga King's Quest et sur le jeu de plateforme Braid, la contribution de Sébastien Genvo (Braid) mobilise habilement les notions narratologiques greimassiennes de pouvoir-faire, vouloir-faire, savoir-faire et devoir-faire dans une approche sémiotique pour analyser la structure du jeu et le lien qu'entretiennent Tim, le héros, et le joueur ou la joueuse.

6 Cette partie se clôt sur un chapitre fort intéressant de Bernard Perron consacré aux modèles féminins présents dans les jeux de type survival horror, dont Resident Evil ou Alone in the dark comptent parmi les principaux représentants. Tout en évoquant prudemment l'existence dans ces jeux d'une « certaine - cette nuance est capitale égalité des sexes » (p. 104), l'auteur conclut "qu'il est difficile pour les personnages féminins de s'éloigner de la catégorie de la "victime", de la figure de la "princesse en détresse" et de "l'idéal de la beauté féminine et de la sexualité" » (p. 115).

7 Si certain-e-s regretteront sûrement de ne pas voir figurer dans cette première partie des contributions portant spécifiquement sur les masculinités, ou s'appuyant sur des données quantitatives (par exemple sur le sexe, le rôle narratif ou les attributs des personnages), on ne peut qu'apprécier la diversité des jeux vidéos étudiés, la variété des ancrages théoriques et le caractère nuancé des conclusions.

8 La seconde partie de l'ouvrage, consacrée aux usages genrés des jeux vidéo par les joueuses et les joueurs, débute par un plaidoyer d'Ivan Mosca pour une utilisation plus fine des catégories de genre, de sexe et de sexualité - trop souvent confondues - dans la recherche articulant genre et jeux vidéo. Insistant sur le potentiel de subversion des normes de genre contenu dans les pratiques, Vanina Mozziconacci s'appuie dans le chapitre 6 sur son expérience des bishōjo games («jeux vidéo de drague » japonais) et des $M M O R P G$ (jeux de rôle en ligne massivement multijoueurs) pour réfléchir à l'impact genré du gameplay et essayer de saisir la complexité des rapports que les joueurs et les joueuses sont susceptibles d'entretenir avec leur(s) avatar(s).

9 Mobilisant avantageusement les concepts de la sociologie interactionniste et de l'ethnométhodologie d'Harold Garfinkel, la contribution de France Vachey propose, à partir d'une observation participante du monde virtuel de World of Warcraft, une réflexion stimulante sur l'exploration des identités de genre permise par le jeu. S'appuyant sur des dizaines de milliers de pages de logs (les scripts des conversations échangées par les joueuses et les joueurs), l'auteure traite successivement des propos généraux, de l'apparence physique, du background et du «corps privé » (p.155) des personnages joueurs pour montrer comment les gameuses et les gamers construisent et interrogent tout à la fois leur identité genrée personnelle par leurs pratiques vidéoludiques.

10 Le chapitre 8, intitulé "Les jeux vidéo au miroir de la télévision - Vers une reconfiguration des stéréotypes de genre? » compare les émissions de télé-crochet des 
années 2000 et 2010 aux jeux vidéo familiaux musicaux (Guitar Hero, $U$ Sing,...) dont elles fournissent le modèle. Tout en rappelant le potentiel immersif de ces jeux, les auteurs soulignent « les facultés de recul de leur public » (p.177) et, à nouveau, l'exploration d'identités de genre alternatives qu'autorise la pratique vidéoludique.

11 La contribution suivante, l'une des plus passionnantes d'un ouvrage qui pourtant n'en manque pas, traite des gamer girls engagées dans la pratique quotidienne et intense d'un MMORPG. S'appuyant sur huit entretiens menés avec des joueuses d'Ever Quest et de World of Warcraft, Catherine Driscoll propose d'étudier «la façon dont le genre informe (et est produit par) l'expérience des joueuses » (p. 182), et esquisse en filigrane toute une typologie des difficultés et des stratégies d'insertion des filles dans les guildes de ces jeux (des collectifs virtuels), largement dominées par les garçons.

12 Dans le chapitre de clôture de cette seconde partie, Catherine Beavis, professeure en sciences de l'éducation, décrit enfin les nombreuses possibilités didactiques offertes en salles de classe par les jeux vidéo - notamment dans les cours de langue - et rappelle "qu'il est possible de respecter les priorités des programmes scolaires [...] tout en y intégrant facilement les jeux vidéo » (p. 241).

D'un point de vue spécifiquement sociologique, on pourrait regretter de ne pas en apprendre davantage sur la position sociale des joueuses et des joueurs interrogée.es ou observé.e.s par les auteur.e.s, et, par extension, de ne pas voir la question du genre plus souvent articulée à celle d'autres rapports de domination tels que la classe ou la race. $\mathrm{Au}$-delà du contenu et des usages des jeux vidéo, la question de la production apparaît par ailleurs comme l'un des angles morts de cet ouvrage. Bien qu'il soit aisé d'imaginer les nombreux obstacles qui s'opposent à la réalisation d'une enquête de ce type (au premier chef desquels le secret commercial), un tel travail livrerait probablement de passionnantes informations sur la manière dont le genre «surgit» dans les contenus vidéoludiques. Toutefois, de par sa richesse et la diversité des approches disciplinaires et méthodologiques sur lesquelles il repose, cet ouvrage comble indéniablement un grand vide dans la production académique spécialisée et ouvre des pistes de recherche particulièrement fécondes pour des travaux à venir.

\section{AUTEURS}

\section{SIMON MASSEI}

Paris 1 Panthéon-Sorbonne (CESSP) 